\title{
ASYMPTOTIC BEHAVIOR OF SOLUTIONS OF A FOURTH ORDER NONLINEAR DIFFERENTIAL EQUATION1
}

\author{
W. E. TAYLOR, JR.
}

\begin{abstract}
In this paper, the asymptotic properties of solutions of a certain fourth order differential equation are considered. Sufficient conditions for oscillation are also given.
\end{abstract}

Introduction. This paper is concerned with the solutions of the differential equation

$$
\left(y^{\prime \prime \prime}+p(x) y\right)^{\prime}+p(x) y^{\prime}+f(y)=0
$$

where $p(x)$ is a continuously differentiable function defined on $[0, \infty)$ satisfying $\int^{\infty} p(x) d x=\infty$. The function $f:(-\infty, \infty) \rightarrow(-\infty, \infty)$ is assumed to be continuous and satisfy the condition $f(y) / y \geqslant m>0$ for $y \neq 0$. Under these assumptions, continuable nontrivial solutions of (1) with a multiple zero are oscillatory. (See Theorem 4.)

The motiviation for this study comes from a recent article by $D$. $L$. Lovelady [2]. In [2], Lovelady considers a special class of nonlinear fourth order equations and derives some oscillation criteria. We also refer to the works of J. W. Heidel [1] and P. Waltman [3] on nonlinear third order differential equations. Unlike the results in [1] and [3], we do not require $p(x)$ to remain one-signed in most of our results.

A solution $y(x)$ of (1) is said to be continuable if it exists on some ray $[a, \infty), a>0$. A nontrivial solution of (1) is oscillatory if it is continuable and has arbitrarily large zeros. By a nonoscillatory solution we mean a continuable solution which is not oscillatory. The term "solution" for the remainder of this work will mean a nontrivial continuable solution.

Main results. Our first result is essential to the results which follow.

LEMMA 1. Let $y(x)$ be a solution of (1). Then

$$
F(y(x))=y(x)\left[y^{\prime \prime \prime}(x)+p(x) y(x)\right]-y^{\prime}(x) y^{\prime \prime}(x)
$$

is nonincreasing on some ray $[a, \infty)$, in fact,

Received by the editors September 9, 1976 and, in revised form, October 16, 1976.

AMS (MOS) subject classifications (1970). Primary 34C10.

Key words and phrases. Zeros, nonlinear oscillations, boundedness.

${ }^{1}$ This research was supported in part by NSF Grant HES 75-03730. 


$$
\{F[y(x)]\}^{\prime}=-\left(y^{\prime \prime}(x)\right)^{2}-y(x) f(y(x)) .
$$

Since $F[y(x)]$ is monotone it follows that $F[y(x)]$ is one-signed on some ray $[c, \infty)$. Using this fact, we will call a solution $u(x)$ of (1), type $\mathbf{I}$, if $F[u(x)] \geqslant 0$ on some ray $[d, \infty)$. If $F[u(c)]<0$ for some $c>0$, then $u(x)$ is said to be type II.

LEMMA 2. Suppose $f(x)$ is a twice continuously differentiable function on $[a, \infty)$ satisfying $\int_{a}^{\infty} f^{\prime \prime}(x) d x<\infty$ and $\int_{a}^{\infty} f^{2}(x) d x<\infty$. Then

$$
\left(\int_{a}^{\infty} f^{\prime 2}(x) d x\right)^{2}<\int_{a}^{\infty}|f|^{2} \cdot \int_{a}^{\infty}\left|f^{\prime \prime}\right|^{2}
$$

Proof. Expand $f$ in a Fourier cosine series on $[a, a+T]$, use Parseval's equality and the C-B-S inequality, then let $T \rightarrow \infty$.

We now examine properties of type I solutions.

THEOREM 3. Let $y(x)$ be a type I solution. Then the following are true:

(i) $\int^{\infty} y^{\prime \prime 2}(x) d x<\infty$ and $\int^{\infty} y(x) f(y(x)) d x<\infty$,

(ii) $\int^{\infty} y^{2}(x) d x<\infty$,

(iii) $\int^{\infty} y^{\prime 2}(x) d x<\infty$.

Proof. Since $y(x)$ is type I, $F[y(x)] \geqslant 0$ on $[a, \infty)$ for some $a>0$. By differentiating $F[y(x)]$ and integrating from $a$ to $x$ we obtain

$$
0 \leqslant F[y(x)]=F[y(a)]-\int_{a}^{x} y^{\prime \prime 2}(t) d t-\int_{a}^{x} y(t) f(y(t)) d t .
$$

This proves (i).

To prove (ii), note that $y(x) f(y(x)) \geqslant m y^{2}(x)$, and apply (i).

Finally, the proof of (iii) follows immediately from (i), (ii) and Lemma 2.

We now consider the type II solutions.

THEOREM 4. Let $y(x)$ be a type II solution. Then $y(x)$ is oscillatory.

Proof. Suppose $y(x)$ is eventually positive, then there exists $x=c$ such that $y(x)>0$ on $[c, \infty)$ and $F[y(c)]<0$.

Consider the function

$$
J(x)=\frac{y^{\prime \prime}(x)}{y(x)}+\int_{c}^{x} p(t) d t .
$$

By differentiating $J(x)$ we find

$$
J^{\prime}(x)=F[y(x)] / y^{2}(x)
$$

for $x>c$. So $J(x)$ is decreasing on $[c, \infty)$. Since $\int_{c}^{\infty} p(x) d x=\infty$, it follows that $y^{\prime \prime}(x)<0$ for large $x$. Since $y(x)>0$ we must have $y^{\prime}(x)>0$ on some ray $[d, \infty), d>c$. The fact that $\int_{c}^{x} p(t) d t \rightarrow \infty$ as $x \rightarrow \infty$ and $J(x)$ is decreasing implies $y^{\prime \prime}(x) / y(x) \rightarrow-\infty$ as $x \rightarrow \infty$. But this implies $y^{\prime \prime}(x)$ is 
bounded away from zero for large $x$, implying $y(x) \rightarrow-\infty$ as $x \rightarrow \infty$. This contradiction proves the theorem.

COROllary. Any nontrivial solution of (1) with a multiple zero is oscillatory.

Lemma 5. Suppose $p(x)>0$ and let $y(x)$ be a type II solution. Then

$$
N[y(x)]=y(x) y^{\prime \prime}(x)-y^{\prime 2}(x) \rightarrow-\infty
$$

as $x \rightarrow \infty$.

Proof. Note that $N^{\prime}[y(x)]=F[y(x)]-p(x) y^{2}(x)<F[y(x)]$. Since $y(x)$ is a type II solution, $F[y(x)]$ is negative and bounded away from zero on some ray $[a, \infty)$ and the result follows.

As our final theorem we list some properties of type II solutions.

THEOREM 6. Let $y(x)$ be a type II solution and assume $p(x)>0$. Then

(i) $y^{\prime}(x)$ is unbounded, and

(ii) $\int_{a}^{\infty} y^{\prime 2}(x) d x=\infty$.

Proof. From Lemma 5, $N[y(x)] \rightarrow-\infty$ as $x \rightarrow \infty$. Since $y(x)$ is oscillatory (Theorem 4) (i) follows immediately by evaluating $N[y(x)]$ along the zeros of $y(x)$.

To prove (ii), integrate $N[y(x)]$ from $c$ to $x$ where $y^{\prime}(c)=0$. Doing so, we obtain

$$
\int_{c}^{x} N[y(t)] d t=y(x) y^{\prime}(x)-2 \int_{c}^{x} y^{\prime 2}(t) d t
$$

But $\int_{c}^{x} N[y(t)] d t \rightarrow-\infty$ as $x \rightarrow \infty$ and, since $y(x)$ is oscillatory, (ii) follows.

\section{REFERENCES}

1. J. W. Heidel, Qualitative behavior of solutions of a third order nonlinear differential equation, Pacific J. Math. 27 (1968), 507-526. MR 39 \# 1738.

2. D. L. Lovelady, An oscillation criterion for a fourth order integrally super linear differential equation, Atti Acad. Naz. Lincei Rend. Cl. Sci. Fis. Mat. Natur. 58 (1975), 532-536.

3. P. Waltman, Oscillation criteria for third order nonlinear differential equations, Pacific $\mathrm{J}$. Math. 18 (1966), 385-388. MR 34 \#422.

Department of Mathematics, Prairie View A \& M University, Prairie View, Texas 77445 